\title{
On the Möbius function of a non-singular binary relation
}

\author{
P. D. Finch
}

Some of the results in the theory of Möbius functions of finite partially ordered sets are extended to arbitrary non-singular binary relations on finite sets.

\section{Introduction}

A systematic theory of inversion formulae on a partially ordered set was developed in Rota [2]. The purpose of this note is to indicate how certain of the key ideas in that theory, for instance Möbius function, incidence algebra and Galois connection, can be discussed in the context of a general binary relation and not just a partial order. For reasons of simplicity we discuss only finite sets and restrict ourselves to just a few instances of results analagous to those established by Rota.

\section{The incidence algebra of a binary relation}

Let $\rho \subseteq S \times S$ be a binary relation on a non-empty finite set $S$. We often write $x \rho y$ instead of $(x, y) \in \rho$. For $(x, y)$ in $S \times S$ we write

$$
[x, y]=\{z: z \in S, x \rho z \& z \rho y\}
$$

and we call $[x, y]$ a $p$-interval of $S$. We note the trivial statements of

LEMMA 2.1 .

(i) $[x, y] \neq \square$ (the empty set) if and only if there is $z$ in $S$ 
with $x \rho z$ and $z \rho y$,

(ii) if $\rho$ is transitive and $[x, y] \neq \square$ then $x p y$,

(iii) if $\rho$ is transitive and reflexive then $[x, y] \neq \square$ whenever $x \rho y$.

We denote the (real) vector space of real-valued functions on $S \times S$ by $A=A(S)$. In fact $A$ is a linear associative algebra with multiplication defined by

$$
f g(x, y)=\sum_{z \in S} f(x, z) g(z, y)
$$

for any $f$ and $g$ in A. The algebra A has a unique identity, namely the Kronecker delta, $\delta(x, y)=1$ or 0 according as $x=y$ or $x \neq y$. We define the zeta-function of $\rho, \zeta=\zeta_{\rho}$ by the equation

$$
\zeta(x, y)= \begin{cases}1 \text { if }(x, y) \in \rho, \\ 0 \text { otherwise. }\end{cases}
$$

We note the obvious

LEMMA 2.2 .

$$
\zeta^{2}(x, y)=\sum_{z \in S} \zeta(x, z) \zeta(z, y)=|[x, y]|
$$

We say that $f$ in $A$ is p-restricted when

$$
(x, y) \notin \rho \Rightarrow f(x, y)=0 \text {. }
$$

Note that the zeta-function is $\rho$-restricted. We write $A_{\rho}=A_{\rho}(S)$ for the subset of $A$ consisting of its $\rho$-restricted elements. The set $A_{\rho}$ is a vector subspace of $A$ but it is not, in general, a subalgebra of A.

LEMMA 2.3. $A_{\rho}$ is a subalgebra of $A$ if and only if $\rho$ is transitive.

Proof. Suppose that $\rho$ is transitive, $(x, y) \notin \rho$ and that $f$ and $g$ are in $A_{\rho}$. Then for any $z$ in $S$ at least one of $(x, z)$ and $(z, y)$ is not in $\rho$ and since $f$ and $g$ are in $A_{\rho}$ we obtain 


$$
f g(x, y)=\sum_{z \in S} f(x, z) g(z, y)=0
$$

that is $f g$ belongs to $A_{\rho}$. Conversely suppose that $A_{p}$ is a subalgebra of $A$, then $\zeta^{2}$ is in $A_{\rho}$ and so, by Lerma 2.2

$$
[x, y] \neq \square \Rightarrow(x, y) \in \rho .
$$

From this implication it follows at once that $\rho$ is transitive.

To facilitate comparison with Rota [2] introduce another binary composition in $A$ by writing

$$
f \circ g(x, y)=\left\{\begin{array}{l}
\sum_{z \in[x, y]} f(x, z) g(z, y), \text { if }(x, y) \in \rho, \\
0, \text { otherwise, }
\end{array}\right.
$$

where we interpret the sum as 0 when $(x, y)$ is in $\rho$ but $[x, y]=\square$. Then $f \circ g$ is in $A_{\rho}$ for each $f$ and $g$ in $A$; in particular this is so whenever $f$ and $g$ are both in $A_{\rho}$. Indeed when $f$ and $g$ belong to $A_{\rho}$ one has

$$
(x, y) \in \rho \Rightarrow f \circ g(x, y)=f g(x, y),
$$

and if, in addition, $\rho$ is transitive then $f g(x, y)=0$ when $(x, y) \notin \rho$ and so, under this additional assumption, $f \circ g=f g$ for any $f$ and $g$ in $A_{\rho}$. Thus when $\rho$ is a partial order, what Rota calls the incidence algebra is just the subalgebra $A_{\rho}$. In the general case, however, $A_{p}$ is not a subalgebra of $A$ and the binary composition ' 0 ' is not associative.

For our purposes it is convenient to work in the algebra $A$ and, although we shall not make further reference to the term, it is that algebra which we would call the incidence algebra of $\rho$.

\section{Non-singular relations}

If $|S|=n$ then each element $f$ of $A$ may be thought of as an $n \times n$ matrix, $\|f(x, y)\|$, the multiplication in $A$ is just matrix 
multiplication and the algebra $A$ is just that of the corresponding algebra of $n \times n$ matrices. We say that the binary relation $\rho$ is non-singular when the matrix $\zeta=\|\zeta(x, y)\|$ is a non-singular matrix, and correspondingly that $\rho$ is singular when the same is true of the matrix $\zeta$. When $\rho$ is non-singular the matrix $\zeta$ has an inverse; we denote it by $\mu=\|\mu(x, y)\|$ and call the corresponding element of $A$ the Möbius function of $\rho$. The well-known Möbius inversion formula is, in this context, nothing more, of course, than a matrix inversion applied to a system of linear equations. Thus if $k$ is a real-valued function on $S$ and

$$
h(y)=\sum_{x:(x, y) \epsilon \rho} k(x)=\sum_{x \in S} k(x) \zeta(x, y)
$$

then, in matrix notation,

$$
h^{\prime}=k^{\prime} \zeta
$$

where $h^{\prime}, k^{\prime}$ are row vectors $(h, k$ being interpreted as column vectors). If $\rho$ is non-singular then $k^{\prime}=h^{\prime} \mu$ where $\mu=\zeta^{-1}$, that is

$$
k(x)=\sum_{y \in S} h(y) \mu(y, x)
$$

This is just the Möbius inversion formula. It is to be noted that when $\rho$ is a partial order the zeta-function is not only invertible but invertible in $A_{\rho}$; this is no longer true in the general case, that is the Möbius function may not be $\rho$-restricted.

Again when $\rho$ is a partial order the Möbius function of a $p$-interval is just the restriction to it of the Möbius function of $\rho$. This result is not generally true; however we note, without proof, the following

LEMMA 3.1. If $\rho$ is non-singular and transitive with Möbius function $\mu$ then the Möbius function of $\rho$ restricted to a $\rho$-interval is the restriction of $\mu$ to that interval.

If $\tilde{\rho}$ is the converse of the relation $\rho$, that is $x \tilde{\rho} y$ when $y \rho x$ then $\tilde{\rho}$ is non-singular when $\rho$ is non-singular and its Möbius function $\tilde{\mu}$ is given by $\tilde{\mu}(x, y)=\mu(y, x)$. 
Finally we remark here, and make use of the fact in Section 5 , that to establish that $\rho$ is non-singular it is sufficient to exhibit a left-inverse $\mu$ of the zeta-function $\zeta$, for $\mu \zeta=\delta$ implies $\zeta \mu=\delta$. This is, of course, obvious in matrix notation; if $A$ and $B$ are $n \times n$ matrices with $A B=I$ (the identity matrix) and $x$ is a vector with $B x=0$ then $x=A B x=0$ and so $B$ is non-singular and $A=B^{-1}$.

\section{GaTois connections}

Let $\rho_{1}, \rho_{2}$ be binary relations on non-empty finite sets $S_{1}, S_{2}$ respectively. Correspondences $x_{1}+x_{1}^{*}$ and $x_{2}+x_{2}^{\dagger}$, from $s_{1}$ to $s_{2}$ and from $S_{2}$ to $S_{1}$ respectively are said to be a Galois connection when

$$
x_{1} \rho_{1} x_{2}^{+} \text {if and only if } x_{2} \rho_{2} x_{1}^{*} \text {. }
$$

When $\rho_{1}, \rho_{2}$ are partial orders this definition of a Galois connection is equivalent to the standard one in, for example, Rota [2]. We prove now our main result; it is

THEOREM 4.1. Let $\rho_{1}, \rho_{2}$ be non-singular binary relations on non-empty finite sets $S_{1}, S_{2}$ respectively, and let $\mu_{1}, \mu_{2}$ be their respective Möbius functions. Suppose that $x_{1} \rightarrow x_{1}^{*}$ and $x_{2} \rightarrow x_{2}^{\dagger}$ is a Galois connection, then for any $x_{1}$ in $S_{1}$ and any $x_{2}$ in $S_{2}$ one has

$$
\sum_{y_{1}: y_{1}^{*}=x_{2}} \mu_{1}\left(x_{1}, y_{1}\right)=\sum_{y_{2}: y_{2}^{\dagger}=x_{1}} \mu_{2}\left(x_{2}, y_{2}\right) .
$$

Proof. Denote the zeta-functions of $\rho_{1}, \rho_{2}$ by $\zeta_{1}, \zeta_{2}$ respectively. Since $y_{1} \rho_{1} y_{2}^{\dagger}$ if and only if $y_{2} \rho_{2} y_{1}^{*}$ we have $\zeta_{2}\left(y_{2}, y_{1}^{*}\right)=\zeta_{1}\left(y_{1}, y_{2}^{\dagger}\right)$ and hence

$$
\sum_{y_{1} \in S_{1}} \mu_{1}\left(x_{1}, y_{1}\right) \zeta_{2}\left(y_{2}, y_{1}^{*}\right)=\delta\left(x_{1}, y_{2}^{+}\right) .
$$

Thus 


$$
\begin{aligned}
\sum_{y_{1}: y_{1}^{*}=x_{2}} \mu_{1}\left(x_{1}, y_{1}\right) & =\sum_{y_{1} \in S_{1}} \mu_{1}\left(x_{1}, y_{1}\right) \delta\left(y_{1}^{*}, x_{2}\right) \\
& =\sum_{y_{1} \in S_{1}} \mu_{1}\left(x_{1}, y_{1}\right) \sum_{y_{2} \in S_{2}} \mu_{2}\left(x_{2}, y_{2}\right) \zeta_{2}\left(y_{2}, y_{1}^{*}\right) \\
& =\sum_{y_{2} \in S_{2}}\left\{\sum_{y_{1} \in S_{1}} \mu_{1}\left(x_{1}, y_{1}\right) \zeta_{2}\left(y_{2}, y_{1}^{*}\right)\right\}_{2}\left(x_{2}, y_{2}\right) \\
& =\sum_{y_{2} \in S_{2}} \delta\left(x_{1}, y_{2}^{\dagger}\right) \mu_{2}\left(x_{2}, y_{2}\right) \\
& =\sum \mu_{2}\left(x_{2}, y_{2}\right) . \\
& y_{2}: y_{2}^{\dagger}=x_{1}
\end{aligned}
$$

This is the desired result.

From this result one recovers Theorem 1 of Rota [2] in the following way. Suppose that $\rho_{1}, \rho_{2}$ are partial orders, and hence non-singular, and that $S_{1}$ has a least element $O_{1}$. Since

$$
o_{1} \rho_{1} x_{2}^{\dagger} \text { if and only if } x_{2} \rho_{2} \circ_{1}^{*}
$$

it follows that $S_{2}$ has a greatest element, $I_{2}=0_{1}^{*}$.

Now suppose, in addition, that the Galois connection has the property

$$
y_{2}^{\dagger}=0_{1} \text { if and only if } y_{2}=0_{1}^{*}
$$

then Theorem 4.1 gives

$$
\mu_{2}\left(x_{2}, I_{2}\right)=\sum_{y_{1}: y_{1}^{*}=x_{2}} \mu_{1}\left(0_{1}, y_{1}\right) \text {, }
$$

and, in particular, when $S_{2}$ also has a least element $\mathrm{O}_{2}$,

$$
\mu_{2}\left(o_{2}, I_{2}\right)=\sum_{y_{1}: y_{1}^{*}=0_{2}} \mu_{1}\left(o_{1}, y_{1}\right) .
$$

This is Rota's Theorem 1.

Replacing $\rho_{2}$ by $\tilde{\rho}_{2}$ one obtains the following dual version of Theorem 4.1.

THEOREM 4.2. Let $\rho_{1}, \rho_{2}$ be non-singular binary relations on non-empty finite sets $S_{1}, S_{2}$ respectively and let $\mu_{1}, \mu_{2}$ be their respective Möbius functions. Suppose that $x_{1} \rightarrow x_{1}^{*}$ and $x_{2} \rightarrow x_{2}^{\dagger}$ are 
correspondences, from $S_{1}$ to $S_{2}$ and $S_{2}$ to $S_{1}$ respectively, such that

$$
x_{1} \rho_{1} x_{2}^{\dagger} \text { if and only if } x_{1}^{*} \rho_{2} x_{2} \text {, }
$$

Then for any $x_{1}$ in $S_{1}$ and any $x_{2}$ in $S_{2}$ one has

$$
\sum_{y_{1}: y_{1}^{*}=x_{2}} \mu_{1}\left(x_{1}, y_{1}\right)=\sum_{y_{2}: y_{2}^{\dagger}=x_{1}} \mu_{2}\left(y_{2}, x_{2}\right) .
$$

\section{Closure operators}

Let $\rho$ be a binary relation on a non-empty finite set $S$. A function $J: S \rightarrow S$ is said to be a $\rho$-closure operator on $S$ when

$$
x \rho J(y) \text { if and only if } J(x) \rho J(y) \text {. }
$$

We note that when $\rho$ is a partial order our definition of a $\rho$-closure operator is equivalent to the standard one in, for instance, Rota [2] where, however, the term closure relation is used. We write

$$
J(S)=\{J(x): x \in S\}
$$

and

$$
\rho_{J}=\rho \cap\{J(S) \times J(S)\} .
$$

If $\rho_{J} \neq \square$ is a binary relation on $J(S)$, we denote its zeta-function by $\zeta_{J}$. We prove

THEOREM 5.1. Let $\rho$ be a non-singular binary relation on a non-empty finite set $S$ and let $J$ be a $\rho$-closure operator. If $\rho_{J}$ is not empty it is non-singular and its Möbius function $\mu_{J}$ is given by

$$
\mu_{J}(a, b)=\sum_{y: J(y)=b} \mu(a, y)
$$

for any $a, b$ in $J(S)$. Moreover for any $b$ in $J(S)$ and any $x$ in $S-J(S)$ one has

$$
y: J(y)=b \quad \mu(x, y)=0 .
$$

Proof. Since $x \rho J(y)$ if and only if $J(x) \rho J(y)$ one has 


$$
\begin{aligned}
\zeta(x, J(y)) & =\zeta_{J}(J(x), J(y)) . \\
\text { Let } a, b \text { and } c \text { be in } J(S) & \text {, then } \\
\sum_{b \in J(S)}\left[\sum_{y: J(y)=b} \mu(a, y)\right] \zeta_{J}(b, c) & =\sum_{b \in J(S)} \sum_{y \in S} \mu(a, y) \delta(J(y), b) \zeta_{J}(b, c) \\
& =\sum_{y \in S} \mu(a, y) \zeta_{J}(J(y), c) \\
& =\sum_{y \in S} \mu(a, y) \zeta(y, c) \\
& =\delta(a, c) .
\end{aligned}
$$

This proves that

$$
\sum_{y: J(y)=b} \mu(a, y)
$$

is a left inverse of $\zeta_{J}$ and hence, because of the remark at the end of Section 3, establishes the first part of the theorem. The second part of the theorem follows from Theorem 4.2 by taking $S_{1}=S, S_{2}=J(S)$, $x_{1}^{*}=J\left(x_{1}\right)$ and $x_{2}^{\dagger}=x_{2}$. Indeed had we been willing to assume the non-singularity of $\rho_{J}$ the first part of the theorem could also have been established by an appeal to Theorem 4.2.

As particular cases of Theorem 5.1 one obtains easily Theorem 4 of Crapo [1] and Proposition 2 of Section 5 of Rota [2].

\section{References}

[1] Henry H. Crapo, "The Möbius function of a lattice", J. Combinatorial Theory 1 (1966), 126-131.

[2] Gian-Carlo Rota, "On the foundations of combinatorial theory I. Theory of Möbius functions", Z. Wahrscheinlichkeits theorie und Verw. Gebiete 2 (1964), 340-368.

Monash University, Clayton, victoria. 